\title{
NORMAL MODE EXPANSION AND STABILITY OF COUETTE FLOW*
}

\author{
BY \\ J. EISENFELD \\ Rensselaer Polytechnic Institute
}

1. Introduction. Chandrasekhar [1] has considered the effect of an axial magnetic field of constant intensity on inviscid Couette flow. He found that the axisymmetric normal mode perturbations satisfying the linearized equations are stable when the angular velocity is increasing in the radial direction. We now consider an arbitrary axisymmetric perturbation and we show that under the above stability condition the perturbation energy is bounded in time. Moreover, the bound can be made arbitrarily small by choosing the initial perturbation values sufficiently small. Hence, the Couette flow is "stable" with respect to axisymmetric perturbations.

The conclusion readily follows once it has been demonstrated that an arbitrary perturbation is a sum of normal modes. The completeness of the normal modes, which is of interest in itself, will be derived from the fact that they are the eigenfunctions of a one to one, linear transformation which is symmetric and completely continuous. This is true even in the absence of the above stability condition.

2. The linearized equations. The linearized equations governing the motion of a perturbation are (cf. [1]):

$$
\begin{gathered}
\left(u_{r}\right)_{t}-2 \Omega u_{\theta}-\frac{\mu H}{4 \pi \rho}\left(h_{r}\right)_{z}=-(\tilde{\omega})_{r}, \\
\left(u_{\theta}\right)_{t}+\left(r \Omega^{\prime}+2 \Omega\right) u_{r}-\frac{\mu H}{4 \pi \rho}\left(h_{\theta}\right)_{z}=0 \\
\left(u_{z}\right)_{t}-\frac{\mu H}{4 \pi \rho}\left(h_{z}\right)_{z}=-(\tilde{\omega})_{z}, \\
\left(h_{r}\right)_{t}-H\left(u_{r}\right)_{z}=0 \\
\left(h_{\theta}\right)_{t}-H\left(u_{\theta}\right)_{z}-r \Omega^{\prime} h_{r}=0, \\
\left(h_{z}\right)_{t}-H\left(u_{z}\right)_{z}=0
\end{gathered}
$$

and the equations of continuity

$$
\left(u_{r}\right)_{r}+u_{r} / r+\left(u_{z}\right)_{z}=0
$$

and

$$
\left(h_{r}\right)+h_{r} / r+\left(h_{z}\right)_{z}=0 .
$$

Here the vectors $u=\left(u_{r}, u_{\theta}, u_{z}\right)$ and $h=\left(h_{r}, h_{\theta}, h_{z}\right)$ denote the perturbations in the radial, transverse, and axial directions of the perturbed velocity and magnetic intensity. $\Omega(r), P(r), \rho, \mu$ and $H$ denote the equilibrium angular velocity, pressure, density, magnetic permeability and magnetic intensity; the notation indicates which

*Received February 23, 1967; revised manuscript received August 16, 1967. 
equilibrium quantities are functions of $r$ and which are constant. In addition, $\tilde{\omega}$ denotes the perturbation of the quantity $P / \rho+\mu H^{2} /(8 \pi \rho)$ and primes denote differentiation with respect to the radial variable $r$, which varies on a finite interval $0<a \leq r \leq b$. The end points correspond to the cylinder walls at which the radial velocity vanishes, i.e.,

$$
u_{r}(a)=u_{r}(b)=0
$$

The axial variable $z$ varies from minus infinity to infinity. The perturbation has finite energy, i.e., it is square integrable.

We consider the equations obtained by taking the Fourier transforms of the perturbation variables. This amounts to replacing the operation of partial differentiation with respect to $z$ by multiplication by $i k$ in (2.1)-(2.8) where $k$ is the wave number. Eliminating $\tilde{\omega}$ between (2.1) and (2.3) we obtain

$$
i k\left(\left(\hat{u}_{r}\right)_{t}-2 \Omega \hat{u}_{\theta}-\frac{i k \mu H}{4 \pi \rho} \hat{h}_{r}\right)=\left(\left(\hat{u}_{s}\right)_{t}-\frac{i k \mu H}{4 \pi \rho}\left(\hat{h}_{z}\right)\right)_{r}
$$

where - denotes Fourier transformation. We see from (2.7) that there is a function $x$ such that

$$
\hat{u}_{r}=i k \chi, \quad \hat{u}_{z}=-\frac{1}{r}(r \chi)_{r} .
$$

Observe from (10.4) that (2.11) is valid at $k=0$. Setting

$$
w_{1}=\chi, \quad w_{2}=-i\left(\frac{\mu}{4 \pi \rho}\right)^{1 / 2} \hat{h}_{\theta},
$$

(2.10) and (1.2) may be written as

$$
\left(\left(D D^{*}-k^{2}\right) w_{1}\right)_{t}+\Omega_{A}^{2}\left(D D^{*}-k^{2}\right) w_{1}-k^{2} \phi w_{1}+2 \Omega \Omega_{A} i k w_{2}=0 .
$$

and

$$
\left(w_{2}\right)_{\mathfrak{l}}+\Omega_{A}^{2} w_{2}+2 \Omega i k \Omega_{A} w_{1}=0
$$

where

$$
\phi=4 \Omega^{2}+r\left(\Omega^{2}\right)^{\prime} \quad \text { and } \quad \Omega_{A}^{2}=\frac{\mu H^{2} k^{2}}{4 \pi \rho}
$$

are Rayleigh's discriminant and the "Alfvén frequency" respectively. Also

$$
D=(\cdot)_{r} \quad \text { and } \quad D^{*}=\frac{1}{r}(r(\cdot))_{r}
$$

are differential operators. Equations (2.4)-(2.8) and (2.11) serve to represent the perturbation variables in terms of $w_{1}$ and $w_{2}$. We now have a $2 \times 2$ system of equations which we can write in the form

$$
(P w)_{t}+\Omega_{A}^{2} P w+S w=0
$$

where

$$
P=\left[\begin{array}{cc}
-\left(D D^{*}-k^{2}\right) & 0 \\
0 & 1
\end{array}\right], \quad S=\left[\begin{array}{cc}
k^{2} \phi & -2 \Omega \Omega_{A} i k \\
2 \Omega \Omega_{A} i k & 0
\end{array}\right]
$$


and the vector $w=\left(w_{1}, w_{2}\right)$ satisfies the boundary condition

$$
w_{1}(a)=w_{1}(b)=0 .
$$

3. The normal modes. The normal modes are those solutions of (2.16) of the form $w_{\sigma}(r) e^{i \sigma t}$. The functions $w_{\sigma}$, also referred to as the normal modes when no confusion arises, are the eigenfunctions of

$$
\lambda P w=S w, \quad w_{1}(a)=w_{1}(b)=0 .
$$

Here we made the substitution

$$
\lambda=\sigma^{2}-\Omega_{A}^{2} .
$$

It will be seen from (4.5) that

$$
D D^{*} y-k^{2} y=0, \quad y(a)=y(b)=0
$$

has no nontrivial solution; hence, its Green's function, $G\left(r, r^{\prime}\right)$, exists. It follows that, $P$ has the inverse

$$
P^{-1}=\left(\begin{array}{ll}
J & 0 \\
0 & 1
\end{array}\right)
$$

where

$$
J(f)=-\int_{a}^{b} G\left(r, r^{\prime}\right) f\left(r^{\prime}\right) d r^{\prime}
$$

is "Green's operator." Eq. (3.1) may now be written as

$$
T w=\lambda w
$$

where $T=P^{-1} S$.

4. The inner product. It will now be shown that there is a symmetric, positive definite, bilinear form $(\cdot, \cdot)_{D}$ for which

$$
(T f, g)_{D}=(f, T g)_{D} .
$$
let

Let $L_{2}$ denote the space of square integrable functions on $(a, b)$. For $f$ and $g$ in $L_{2}$

$$
(f, g)_{r}=\int_{a}^{b} f \bar{g} r d r,
$$

where bars denote complex conjugation. Upon integrating by parts one sees that $D D^{*}$ is symmetric with respect to (4.2) for functions vanishing at the end-points. It follows that $J$ is also symmetric. Let

$$
D_{P}=\left\{f: D^{2} f_{1}, f_{2} \varepsilon L_{2}, f_{1}(a)=f_{1}(b)=0\right\} .
$$

Then on $D_{P},(P f, g)$ where

$$
(f, g)=\left(f_{1}, g_{1}\right)_{r}+\left(f_{2}, g_{2}\right)_{r} .
$$

Since, after integrating by parts,

$$
\left(-\left(D D^{*}-k^{2}\right) f, f\right)_{r}=\int_{a}^{b}\left(\left|D^{*} f\right|^{2}+k^{2}|f|^{2}\right) r d r>0,
$$


we see that the inner product

$$
(f, g)_{P}=(P f, g)_{r}
$$

is symmetric and positive definite on $D_{P}$. Moreover, $S$ is symmetric with respect to (4.4); hence,

$$
(T f, g)_{P}=\left(P P^{-1} S f, g\right)_{r}=(S f, g)_{r}=(f, S g)_{r}=\left(f, P P^{-1} S g\right)_{r}=(f, T g)_{P} \quad \text { on } \quad D_{P} .
$$

5. Complete continuity. With respect to the norm,

$$
\|w\|_{P}=(w, w)_{P}^{1 / 2}=\left(\left\|D^{*} w_{1}\right\|_{r}^{2}+k^{2}\left\|w_{1}\right\|_{r}^{2}+\left\|w_{2}\right\|_{r}^{2}\right)^{1 / 2}
$$

where $\|\cdot\|_{r}=(\cdot, \cdot)_{r}^{1 / 2}, D_{P}$ is a pre-Hilbert space. Since

$$
a\|\cdot\|_{2}^{2} \leq\|\cdot\|_{r}^{2} \leq b\|\cdot\|_{2}^{2}
$$

we see that $\|\cdot\|_{r}$ is equivalent to the $L_{2}$ norm, $\|\cdot\|_{2}$. Moreover, $D^{*} f$ is in $L_{2}$ if and only if $D f$ is in $L_{2}$. Therefore, the completion of $D_{P}, H$, is precisely the set of functions in the product $S_{2}^{1} \times L_{2}$ where $S_{2}^{1}$ is the Sobolev space of functions which are continuous on $(a, b)$, vanish at the end points and have "strong" first derivatives in $L_{2}$ (cf. [6]). To see that $T$ is completely continuous on $H$ we compute

$$
\|T w\|_{p}=\left[(J v, v)_{r}+4 \Omega_{A}^{2} k^{2}\left\|\Omega w_{1}\right\|_{r}^{2}\right]^{1 / 2}
$$

where $v=k^{2} \phi w_{1}-2 \Omega \Omega_{A} i k w_{2}$ and $J$ is given by (3.4). We wish to show that if $w(n)$ is a bounded sequence in $H$ then there is a subsequence of integers for which $\| T(w(n)-$ $w(m)) \|_{p} \rightarrow 0$ as $n, m \rightarrow \infty$. Since $J$ is an integral operator with a bounded kernel, it is completely continuous on $L_{2}$. Since the sequence $v(n)$ is bounded in $L_{2}, J v(n)$ has a Cauchy subsequence in $L_{2}$. Therefore $|J(v(m)-v(m)), v(n)-v(m)| \leq|| J v(m)-$ $J v(n)\|\| v(m)-v(n) \| \rightarrow 0$ as $n, m \rightarrow \infty$ on a subsequence of integers. We may now assume that the subsequence is the original sequence. In view of (5.2) it remains to show that $\left\|w_{1}(n)\right\|_{r}$ has a Cauchy subsequence, but this is a special case of Rellich's Theorem (cf. $[5, \mathrm{p} .30])$. It also may be deduced from the fact that $w_{1}(n)$ is the image of the completely continuous operator, $\left(D^{*}\right)^{-1}=r \int_{a}^{r}(\cdot) s^{-1} d s$ and that $D^{*} w_{1}(n)$ is bounded in $L_{2}$.

6. Differentiability of the eigenfunctions. It will be shown that in the process of "completing" $D_{P}$ no new eigenfunctions are added. In fact, it will be seen that the eigenfunctions are as differentiable as the equilibrium angular velocity, and that they vanish at the end points. Suppose that $w$ is an eigenfunction. This means that

$$
J\left(k^{2} \phi w_{1}-2 \Omega \Omega_{A} i k w_{2}\right)=\lambda w_{1}
$$

and

$$
2 \Omega \Omega_{A} i k w_{1}=\lambda w_{2} .
$$

Since $w_{1}$ is continuous and vanishes at the end points the same is true of $w_{2}$ by (6.2). In view of (6.1) $w_{1}$ has two continuous derivates since the kernel of $J$ is the Green's function of a second order differential equation. Now if $\Omega$ is twice differentiable, the same is true of $w_{2}$. Repeating this argument as many times as necessary we see that $w$ is as differentiable as $\Omega$.

We see also from (6.1) and (6.2) that if $\lambda=0$ then $w_{1}=0$ and $J\left(\Omega w_{2}\right)=0$. Applying $\left(D D^{*}-k^{2}\right)$ to the latter gives $w_{2}=0$. Here we are assuming that $\Omega$ does not vanish identically in any subinterval. It follows that zero is not an eigenvalue, i.e., $T$ is one to one. 
7. The completeness theorem. It has been shown in the preceding sections that the normal modes are the eigenfunctions of a symmetric, completely continuous, one to one, linear transformation of the complete Hilbert space of vectors, $w=\left(w_{1}, w_{2}\right)$ such that $w_{1}$, its derivative, $D w_{1}$, and $w_{2}$ are square integrable on $(a, b)$. Convergence in the space is taken in the sense of (5.1). If follows (cf. [4]) that for any vector $f$ in the space

$$
f=\sum_{n}\left(f, w_{n}\right)_{P} w_{n}
$$

where the normal modes $w_{n}$ are normalized by $\left\|w_{n}\right\|_{P}=1$. Moreover, the normal modes are orthonormal, i.e.,

$$
\left(w_{n}, w_{m}\right)_{P}=\delta_{n m} .
$$

8. Distribution of the characteristic frequencies. We now assume the stability condition (cf. Sec. 1):

$$
\left(\left(\Omega^{2}(r)\right)\right)^{\prime}>0 .
$$

It will be shown that the characteristic frequencies $\sigma_{n}$, which are related to the eigenvalues $\lambda_{n}$ by (cf. (3.2))

$$
\lambda_{n}=\sigma_{n}^{2}-\Omega_{A}^{2},
$$

are real, nonzero, $\neq \pm \Omega_{A}^{2}$, but tend to $\pm \Omega_{A}^{2}$ as $n \rightarrow \infty$. The last two properties are true in the absence of (8.1) and follow immediately from the fact that $\lambda_{n} \rightarrow 0$ as $n \rightarrow \infty$ and that $\lambda_{n} \neq 0$, since they are the eigenvalues of an operator with properties stated in Sec. 7. The first two properties follow from the relation:

$$
\sigma_{n}^{2} / \Omega_{A}^{2}>\int_{a}^{b} r\left(\Omega^{2}\right)^{\prime}\left|w_{1}\right|^{2} r d r / \int_{a}^{b} \phi\left|w_{1}\right|^{2} r d r
$$

where $w_{1}$ is the first component of the $n$th normal mode. To verify (8.3) observe that by substituting for $w_{2}$ from (6.2) into (6.1) and then applying $D D^{*}-k^{2}$ to both sides $w_{1}$ satisfies

$$
D D^{*} w_{1}+k^{2}\left(4 \Omega^{2} \Omega_{A}^{2} \lambda_{n}^{-2}+\phi \lambda_{n}^{-1}-1\right) w_{1}=0 .
$$

Multiplying both sides of (8.4) by $r \bar{w}_{1}$, integrating from $a$ to $b$ and then integrating by parts using (3.1), we obtain

$$
A \lambda_{n}^{-2}+B \lambda_{n}^{-1}=C
$$

where

$$
\begin{aligned}
& A=4 k^{2} \Omega_{A}^{2} \int_{a}^{b} \Omega^{2}\left|w_{1}\right|^{2} r d r>0, \\
& B=k^{2} \int_{a}^{b} \phi(r)\left|w_{1}\right|^{2} r d r>0
\end{aligned}
$$

and

$$
C=k^{2} \int_{a}^{b}\left|w_{1}\right|^{2} r d r+\int_{a}^{b}\left|D^{*} w_{1}\right|^{2} r d r>0 .
$$


Since $C$ is positive, $A \lambda_{n}^{-2}+B \lambda_{n}^{-1}>0$. Assuming $\lambda_{n}$ negative, this is equivalent to (cf. (2.15)) $-1-\lambda_{n}^{-1} \Omega_{A}^{2}>\beta$ where

$$
\beta=\int_{a}^{b} r\left(\Omega^{2}\right)^{\prime}\left|w_{1}\right|^{2} r d r / \int_{a}^{b} 4 \Omega^{2}\left|w_{1}\right|^{2} r d r .
$$

Substituting now from (8.2) yields $\sigma_{n}^{2}>\Omega_{A}^{2} \beta /(1+\beta)$ and this is equivalent to (8.3). When $\lambda_{n}$ is positive (8.3) is immediate since the right side of (8.3) is less than one.

9. Normal mode expansion. Consider an arbitrary perturbation and let $w$ be defined by (2.12). Since $w$ is in $D_{P}$ for each $t$ we have from (7.1) the expansion

$$
w=\sum_{n} \alpha_{n} w_{n}
$$

where the Fourier coefficient

$$
\alpha_{n}=\left(w, w_{n}\right)_{P}
$$

is a function of $t$. To determine this dependence on time we obtain from (3.2) and (3.5) that $\alpha_{n}$ satisfies $\left(\alpha_{n}\right)_{u t}+\sigma_{n}^{2} \alpha_{n}=0$ and hence there are constant $A_{n}$ and $B_{n}$ such that

$$
\alpha_{n}(t)=A_{n} \cos \left(\sigma_{n} t\right)+B_{n} \sin \left(\sigma_{n} t\right) .
$$

One finds from (9.3) that

$$
A_{n}=\alpha_{n}(0), \quad B_{n}=\left(\alpha_{n}(0)\right)_{\imath} / \sigma_{n}
$$

and hence

$$
\alpha_{n}(t)=\alpha_{n}(0) \cos \left(\alpha_{n} t\right)+\left(\alpha_{n}(0)\right)_{\imath} \sin \left(\alpha_{n} t\right) / \sigma_{n} .
$$

Equations (9.1) and (9.4) yield a normal mode expansion for $w$. Using (2.11) and (2.12) and Fourier inverting one obtains a normal mode expansion for $u_{s}, u_{s}$ and $h$. Normal mode expansion for the remaining perturbation variables can be obtained from (2.1)(2.8).

10. Stability. We now consider

$$
I=\int_{a}^{b} \int_{-\infty}^{\infty}\left(\left|u_{r}\right|^{2}+\left|u_{z}\right|^{2}+\frac{\mu}{4 \pi \rho}\left|h_{\theta}\right|^{2}\right) d z r d r
$$

which represents part of the perturbation energy. We wish to show that $I$ is bounded in time and that the bound depends only on the perturbation values at $t=0$. Using Parseval's formula for Fourier transforms we find, using (2.11), (2.12) and (5.1), that $I=\int_{-\infty}^{\infty}\|w\|_{P}^{2} d k$. Using Parseval's formula now on the expansion (9.1) we have that $I=\int_{-\infty}^{\infty}\left(\Sigma_{n}\left|\alpha_{n}\right|^{2}\right) d k$. It follows from (9.4) that $I(t) \leq I(0)+J$ where

$$
J=\int_{-\infty}^{\infty}\left(\sum_{n}\left|\alpha_{n}(0)_{t} / \sigma_{n}\right|^{2}\right) d k .
$$

The characteristic values $\sigma_{n}$ depend on $k$; however, it follows from (8.3) that $\sigma_{n}^{2}>\gamma k^{2}$ where the constant

$$
\gamma=\left(\mu H^{2} / 4 \pi \rho\right) \min \left(r\left(\Omega^{2}\right)^{\prime} / \phi\right)
$$

is positive by (8.1). It remains to show that

$$
\int_{-\infty}^{\infty}\left(\sum_{n} \mid\left(\alpha_{n}(0)_{\imath} /\left.k\right|^{2}\right) d k<\infty .\right.
$$


We have from (2.7) and (2.9) that

$$
\hat{u}_{r}=\frac{-i k}{r} \int_{a}^{r} \hat{u}_{z} r^{\prime} d r^{\prime}
$$

and from (2.8) that

$$
\hat{h}_{r}=\frac{-i k}{r} \int_{a}^{r} \hat{h}_{z} r^{\prime} d r^{\prime}
$$

provided

$$
\hat{h}_{r}(a, k, 0)=0 .
$$

We know that $(h(a, k, t))_{t}=0$ by $(2.4)$, but (10.6) is not true in general. However, for the sake of showing stability, we can subtract the stable perturbation $u_{r}=u_{0}=u_{s}$ $h_{s}=0, h_{r}=h_{r}(a, z, 0)$ from the perturbation under the consideration and hence assume, without loss of generality, that (10.6) is true. It now follows from Parseval's formula and equations (2.1)-(2.6), (10.4), (10.5) that the left side of (10.3) is

$$
\int_{0}^{b} \int_{-\infty}^{\infty}\left(\left|\frac{\mu H}{4 \pi \rho} h_{z}-\tilde{\omega}\right|^{2}+\left|\frac{1}{r} \int_{a}^{r}\left(u_{z}\right)_{t} r d r^{\prime}\right|^{2}+\frac{\mu}{4 \pi \rho}\left|H u_{\theta}-\Omega^{\prime} \int_{a}^{r} h_{s} r^{\prime} d r^{\prime}\right|^{2}\right) d z r d r
$$

evaluated at $t=0$. We have thus shown that the partial perturbation energy represented by (10.1) is bounded, the bound depending on the initial perturbation values.

The remaining perturbation variables are treated similarly. We observe from (2.8) that there is a function $\tilde{\chi}$ such that

$$
\hat{h}_{r}=i k \tilde{\chi}, \quad \hat{h}_{z}=-\frac{1}{r}(r \tilde{\chi}) .
$$

Setting

$$
Z_{1}=\tilde{\chi}, \quad Z_{2}=\frac{-(4 \Omega \rho)^{1 / 2} i}{\mu^{1 / 2} H}\left(H \hat{u}_{\theta}+\left(r \Omega^{\prime}+2 \Omega\right) \tilde{\chi}\right)
$$

we find that $Z$ is a solution of (2.13)-(2.14). Therefore the above inequalities relating $w$ to its initial values apply equally as well to $Z$ except that (10.8) replaces (2.12). If we let

$$
M=\int_{a}^{b} \int_{-\infty}^{\infty}\left(\left|h_{r}\right|^{2}+\left|h_{z}\right|^{2}+\frac{4 \pi \rho}{\mu H^{2}}\left|H u_{\theta}-\frac{\left(r \Omega^{\prime}+2 \Omega\right)}{r} \int_{a}^{b} h_{z} r^{\prime} d r^{\prime}\right|^{2}\right) d z r d r .
$$

then

$$
M(t) \leq M(0)+(H / \gamma) I(0)
$$

where $\gamma$ and $I$ are given by (10.2) and (10.1). It follows from (10.9) and from a similar bound on $I$ calculated above that the energy norm,

$$
\int_{a}^{b} \int_{-\infty}^{\infty}\left(\left|u_{r}\right|^{2}+\left|u_{\theta}\right|^{2}+\left|u_{z}\right|^{2}+\left|h_{r}\right|^{2}+\left|h_{\theta}\right|^{2}+\left|h_{z}\right|^{2}\right) d z d r,
$$

is bounded by some other norm evaluated at $t=0$. In this sense the perturbation is stable.

11. Discussion. A somewhat more complete version may be found in the author's 
dissertation [3]. There it is shown that the expansion formula converges pointwise as well as in the mean. Asymptotic approximations are obtained for the normal modes and the characteristic frequencies. It is also shown that the stability condition (8.1) is necessary as well as sufficient in the sense that if the angular velocity is decreasing in some subinterval and the magnetic intensity is sufficiently small then unstable normal modes do exist.

The case of the nonaxisymmetric perturbation still waits to be treated.

The theory of eigenfunction expansions has suffered neglect in the last decades. The study presented here promises to be useful in extending some results of Langer.

\section{ReFERENCES}

1. S. Chandrasekhar, Hydrodynamic and hydromagnetic stability, Oxford University Press, England, 1961

2. E. A. Coddington and N. Levinson, Theory of ordinary differential equations, McGraw-Hill, New York, 1955

3. J. Eisenfeld, Completeness theorems in hydrodynamic stability, Ph.D. Thesis, University of Chicago, Chicago, Ill., 1966

4. F. Riesz and B. Sz. Nagy, Functional analysis, Ungar, New York, 1955

5. S. Agmon, Elliptic boundary value problems, Van Nostrand, Princeton, N. J., 1965

6. S. G. Mikhlin, Variational methods in mathematical physics, Macmillan, New York, 1964

The research reported here was supported in part by the U. S. Air Force Office of Scientific Research under grant AF-AFOSR-712-65 with the University of Chicago. This research is an extension of part of the author's doctoral thesis under the guidance of Professor Lebovitz at the University of Chicago. 